\title{
Rheological behaviour of pyroclastic debris flow
}

\author{
A. M. Pellegrino ${ }^{1}$, A. Scotto di Santolo ${ }^{1}$, A. Evangelista ${ }^{1}$ \\ \& P. Coussot ${ }^{2}$ \\ ${ }^{I}$ Department of Hydraulic, Geotechnical and Environmental \\ Engineering, University of Naples "Federico II", Italy \\ ${ }^{2}$ Université Paris - Est, Institut Navier, France
}

\begin{abstract}
The pyroclastic soils that cover the mountains of the Campania region in Italy are usually unsaturated and collapse due to rainfall infiltration triggering landslides. The evolution of these soils after collapse is not well understood. Indeed, their post-failure behaviour may be "solid-like" or "fluid-like", depending on causes that are not well known. The objective of this paper is the study carried out on the rheological behaviour of the "fluid-like" pyroclastic material with fluid mechanics tools: a vane rotor rheometer and an inclined plane. Two natural pyroclastic deposits have been sampled and different soils-water mixtures have been analysed. The main results have been explained and discussed
\end{abstract}

Keywords: debris flow, pyroclastic soil, solid-liquid transition, rheology, laboratory activity, fluids model, yield stress, critical shear rate.

\section{Introduction}

The Campania region has been covered by pyroclastic deposits generated by different volcanic centres, the most famous of which is the Somma-Vesuvius, which is still active inside the so-called Campanian Volcanic Zone. In this area, pyroclastic soils (mostly ash and pumices) and soft rocks (tuff) have been extensively used since antiquity for construction purposes. The cover is cohesionless and poses severe slope stability problems. The landslides have been classified as translational or rotational sliding or falls that lead to debris flows. As a result of the ceaseless growth and spreading of urbanised areas and 
infrastructures, the risk of landslides have been increased enormously, as testified by hundreds of victims of flowslides in the last fifty years [1, 2].

Despite the relevance of the problem, a comprehensive geotechnical classification of these deposits is still lacking. While the mechanical properties of the Campanian natural soils are the object of numerous studies, the postfailure behaviour of the material may be "solid-like" or "fluid-like" according to causes that are not well known. This paper has been focused on the post-failure behaviour of such materials which it seems to depend on several factors (i.e., the geometry of the slope, the sequence and mechanical properties of the cover, the porosity, the grain size distribution, the stress conditions and the characteristics of the rainfall prior to and following the triggering) $[3,4]$.

As an idealisation, a debris flow has been often considered as a mixture of viscous slurry, made up of the finer grain sizes and water, and coarse particles. In general, fluid-to-fluid, fluid-to-solid, and solid-to-solid interactions can play an important role [5]. Some researchers have been used such codes for backanalysing debris flows in the Campania region [6-8]. Alternatively, the use of a fluid mechanical treatments to study the rheological behaviour of these pyroclastic deposits remixed with water has not been studied often because of the flow curve (the relationship between shear stress and shear rate in the steady state) is nevertheless not so easy to evaluate. Scotto di Santolo [3, 4] and Scotto di Santolo et al. [9] have carried out preliminary rheometrical tests on fine particle-water suspensions of some pyroclastic soils collected in Campania region at different solid volumetric concentrations; they have found that the mixture behaves as a non-Newtonian fluid with a yield stress and that the behaviour varying with the solid concentration.

In this paper, the further results of a more complete laboratory activity on two of these pyroclastic deposits have been reported in order to evaluate whether the rheological approach can be a useful tool for understanding in which condition the pyroclastic soil changes behaviour from that of a soil to that of a fluid.

\section{Materials}

The materials tested have been collected from the source area of two debris flows in the Campania region [9]. Material I has been sampled in Nocera, Salerno (March 2005) and material II has been sampled in Monteforte Irpino, Avellino (May 1998). The soil type, in a thickness of about a metre, depends on the most recent pyroclastic deposits deriving from the volcanic activity of Mount Somma/Vesuvius [3, 4, 10]. The main grain size distributions of the collected samples are reported in fig. 1 . Soil I and soil II are sandy silt with a small clay fraction. The bedrock underlying the soil is limestone for materials I and II. Mean physical properties are reported in Table 1 (where $G_{S}$ is the specific gravity of soil particles, $\gamma_{\mathrm{d}}$ and $\gamma$ are the dry and total weight of soil per unit volume respectively, $\mathrm{n}$ is the porosity, and $\mathrm{S}_{\mathrm{r}}$ is the degree of saturation).

Due to the size of the rheometrical facilities [11], the soil fraction with a particle diameter less than $0.5 \mathrm{~mm}$ has been kept. This represents about $70 \%$ of the whole grain size distribution (see fig. 1), so it has been expected that the behaviour of this material represents the behaviour of the full mixture well. 


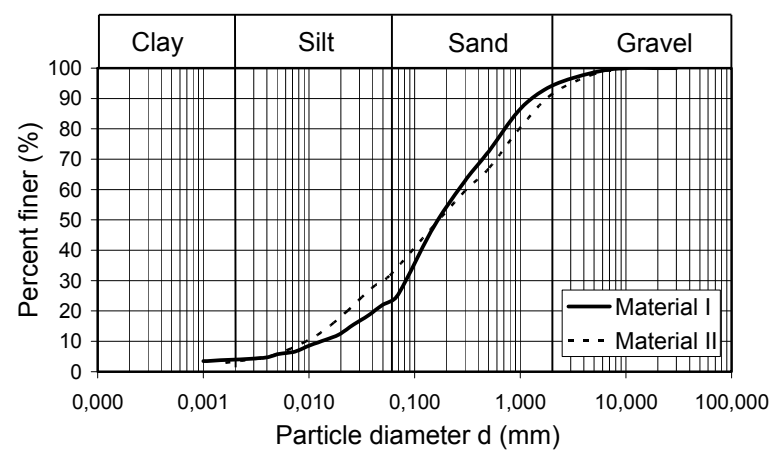

Figure 1: Grain size distribution of the natural deposits analysed.

Table 1: $\quad$ Main physical properties of the tested debris flow materials.

\begin{tabular}{|c|c|c|c|c|c|c|c|}
\hline Debris flow Site & Substratum & Material & $\begin{array}{c}\mathrm{G}_{\mathrm{S}} \\
(1)\end{array}$ & $\begin{array}{c}\gamma_{\mathrm{d}} \\
\left(\mathrm{kN} / \mathrm{m}^{3}\right)\end{array}$ & $\begin{array}{c}\gamma \\
\left(\mathrm{kN} / \mathrm{m}^{3}\right)\end{array}$ & $\begin{array}{c}\mathrm{n} \\
(1)\end{array}$ & $\begin{array}{c}\mathrm{S}_{\mathrm{r}} \\
(1)\end{array}$ \\
\hline Nocera (SA) & Carbonatic & $\mathrm{I}$ & 2.61 & 9.08 & 11.35 & 0.66 & 0.35 \\
\hline Monteforte Irpino (AV) & Carbonatic & II & 2.57 & 7.11 & 12.11 & 0.71 & 0.71 \\
\hline
\end{tabular}

All experiments have been carried out with mixtures of different water contents in order to consider a significant range of the sediment concentrations for each material tested (according to the in situ porosity; see Table 1). The solid volumetric concentration, i.e., the ratio of the volume of solids to the total volume (water plus solids), has been used and it has been defined as:

$$
\Phi=\frac{\mathrm{V}_{\mathrm{s}}}{\mathrm{V}_{\mathrm{w}}+\mathrm{V}_{\mathrm{S}}}
$$

where $\mathrm{V}_{\mathrm{w}}$ and $\mathrm{V}_{\mathrm{s}}$ are, respectively, the volumes of water and solid in the sample. For each material tested, material mixtures of about $500 \mathrm{ml}$ have been prepared, mixing soils and water with an electronic mixer for 15 minutes. Then a sample volume of about $30 \mathrm{ml}$ for each test has been used at a constant temperature $\left(23^{\circ} \mathrm{C}\right)$.

\section{Set up and procedures}

The behaviour of the material mixtures analysed has been investigated with two experimental apparatuses: a conventional rotational rheometer and an inclined plane.

\subsection{Rheometer}

A rotational rheometer CVOR (Bohlin Instruments) equipped with a vane rotor geometry system (fig. 5a) has been used. It consists of four thin blades arranged at equal angles around a small cylindrical shaft: the blade radius was $13 \mathrm{~mm}$, and the blade height was $48 \mathrm{~mm}$. The vane rotor has been immersed in the sample contained in a cylindrical cup $18.5 \mathrm{~mm}$ in radius. The rotor has been rotated 
around its axis at a given rotational speed $\Omega$, and the torque $T$ has been measured. Under usual assumptions (no inertia effects, negligible normal stress differences) the shear stress and the shear rate within the material are given as:

$$
\begin{gathered}
\dot{\gamma}=\frac{\Omega \mathrm{R}_{1}}{\mathrm{R}_{2} \mathrm{R}_{1}} \\
\tau=\frac{\mathrm{T}}{2 \pi \mathrm{R}_{1}^{2} \mathrm{~L}}
\end{gathered}
$$

where $R_{1}$ and $R_{2}$ are, respectively, the blade radius and the cup radius, and $L$ is the material depth. Two kinds of tests have been carried out: creep and stress sweep. During the creep test, a constant torque (associated with a constant stress) has been imposed and the material behaviour has been monitored from the resulting deformation versus time curve. Deformation is expressed in terms of angle of creep (i.e., the angle, in radians, of the displacement after the creep stress was applied). During a stress sweep, the flow curves have been determined by applying an increasing-decreasing shear stress ramp. In that case, the material response has been followed from the resulting shear stress - shear rate curve. Before each test, a pre-shear has been applied, which consists of imposing a very rapid flow and then leaving the material at rest for some time in order to provide a homogeneous state of the sample before carrying out each test defined.

\subsection{Inclined plane}

The inclined plane test consists of leaving a certain amount of paste over an inclined plane and analysing the fluid depth profile in rheological terms. The equipment for the test consists of a roughness plane and an inclinometer (fig. b). The test procedure is the following: first the mixtures have been spilled on
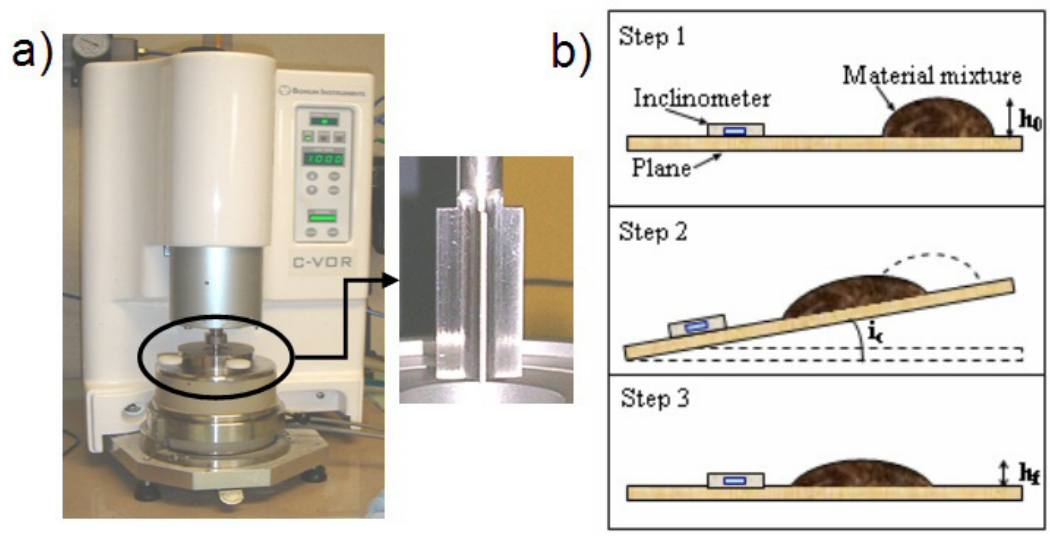

Figure 2: Experimental apparatus used: a) the rotational rheometer (CVOR Bohlin) and the vane rotor geometric system; b) scheme of the inclined plane test procedure. 
the plane (step 1 in fig. 2b) and with a graduated rule the initial thickness of the deposits has been measured, $\mathrm{h}_{0}$, as the averaged value of several measurements. Then, the plane has been inclined and stopped it when the front of the deposit started to move (step 2 in fig. 2b). At that moment, the critical angle has been defined, $i_{c}$, as the corresponding inclination of the plane. Finally the final thickness of the deposits, $\mathrm{h}_{\mathrm{f}}$, has been defined as the averaged value (step 3 in fig. 2b). Under the usual so-called "lubrication assumption" [12], a simple momentum balance provides the shear stress distribution within the material and the critical thickness may be used for determining two critical stresses: a "static" yield stress $\left(\tau_{\mathrm{c} 1}\right)$ and a "dynamic" yield stress $\left(\tau_{\mathrm{c} 2}\right)$ :

$$
\begin{gathered}
\tau_{C 1}=\rho g h_{0} \sin \left(i_{c}\right) \\
\tau_{\mathrm{C} 2}=\rho g h_{\mathrm{f}} \sin \left(i_{\mathrm{c}}\right)
\end{gathered}
$$

where $\rho$ is the mixture density and $g$ is the acceleration of gravity.

\section{Behaviour evolution with the solid fraction}

The first step with the selected materials was to investigate their overall mechanical behaviour as a function of the solid concentration. By mixing the solid with water, a mixture has been obtained that was in three possible states:

- For sufficiently low volume fractions, the solid particles rapidly (within a few seconds) settle down, leading to an apparent phase separation; such a situation follows from the fact that the suspended particles do not interact when they are dispersed in water (such as in the very first seconds after preparation) so that we are dealing with a Newtonian fluid with an apparent viscosity typically of the order of ten times that of pure water; when the particles have settled, we are no longer dealing with a homogeneous material, and nothing can be said about its viscosity.

- For too high volume fractions, the suspension obtained is in fact a kind of paste of high strength, which easily breaks like a solid when it is deformed; such a material cannot be considered as a fluid able to undergo reversible large deformations without changing its basic properties.

- For intermediate volume fractions, we can observe some slight sedimentation after significantly longer times of rest, a point that we will discuss below; the material thus remains homogeneous over a reasonable time of observation and can flow like a liquid.

The lower bound is $32 \%$ for material I and $30 \%$ for material II. These values were very low for each material tested, slightly different from each other but in agreement with the porosity of the natural deposits (shown in table 1). The upper bound of solid concentration is $42 \%$ for material I and $38 \%$ for material II. For higher solid volumetric concentrations, no steady flow regime occurred.

Surprisingly, the range of solid concentrations in which the material mixtures can be considered as "fluid-like" is rather narrow for each material tested, in contrast with clay-water systems, for example, for which one may get homogeneous fluids in a range from one percent to several tens of percents. 
Subsequently the "fluid-like" behaviour within these intervals identified for each material has been investigated.

\section{Rheometer results}

\subsection{Creep test results}

The results of creep tests conducted on material I mixtures at two different volumetric solid concentrations are reported in fig. 3. For stresses lower than the critical value the curve remains concave with a slope continuously decreasing in time and exhibits an apparent horizontal asymptote: no steady state has been reached and the material apparently stops moving. This should correspond to the solid regime of the material. For stress values higher than the critical value the initial slope of the curve is similar to that under smaller stresses, but after some time, there is an inflection point, and the curves tend to reach an inclined straight line gradually with a slope equal to 1: a steady state flow has been reached. These curves correspond to the liquid regime of the material: the deformation trend is linear with time and the material shows a constant shear rate when a constant shear stress is applied. In this context, this critical value of stress has been defined the static yield stress, $\tau_{\mathrm{cl}}$, which is the value of stress at which the material ultimately flows in a liquid regime. When looking at the set of creep curves for different stress values, different aspects have been seen. In some cases (see fig. 3a), there is apparently a smooth transition from the solid to the liquid regime. Such behaviour corresponds to simple yield stress behaviour: around the yield stress, the material changes from a situation in which it does not flow (solid regime) to a situation in which it flows extremely slowly (liquid regime just above the yield stress). In other cases, the transition from the solid to the liquid regime is more abrupt (see fig. $3 b$ ): around the yield stress the material changes from not flowing to flowing at a relatively high shear rate.
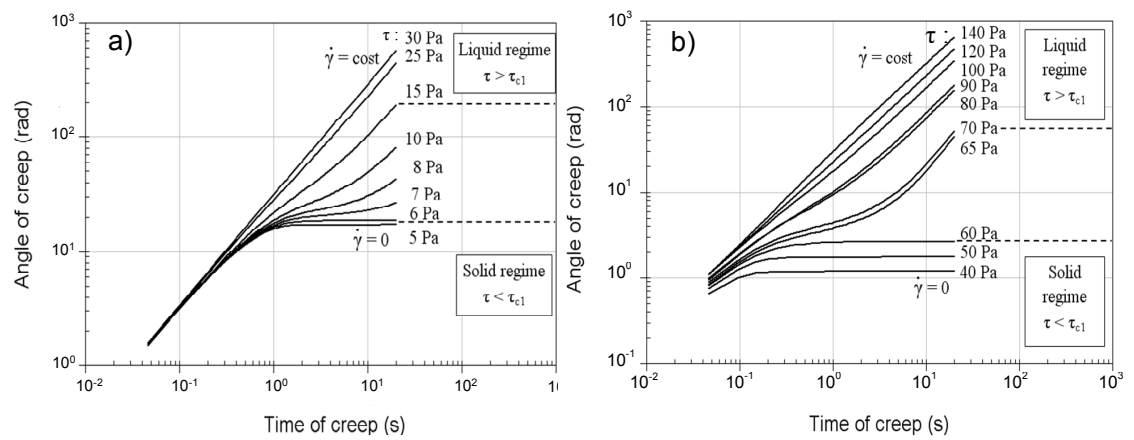

Figure 3: $\quad$ Creep curves for different stress values of material I at: a) solid concentration of $35 \%$ and $b$ ) solid concentration of $40 \%$. 

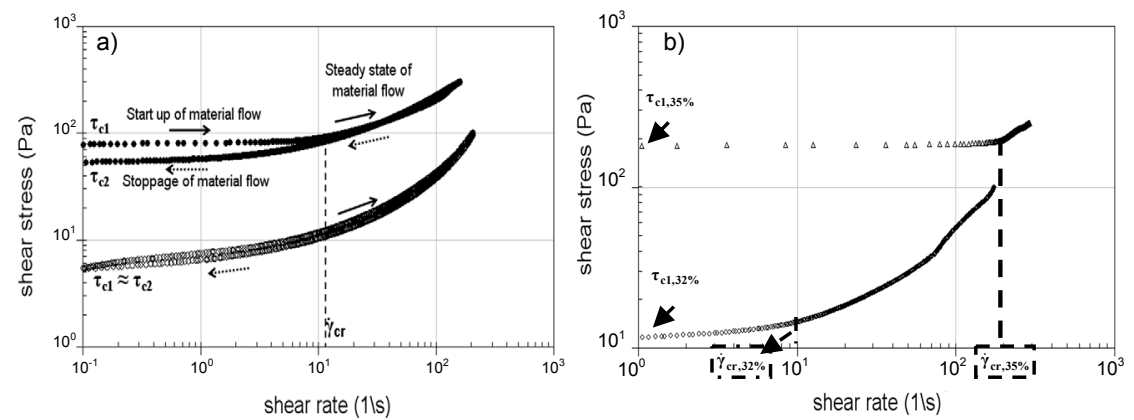

Figure 4: a) Flow curves of material I mixtures at two different solid concentrations obtained with an increasing stress ramp (arrow with continuous line) and a decreasing stress ramp (arrow with dashed line). The filled circles are the flow curves at a solid concentration equal to $40 \%$, while the empty circles are the flow curves at a solid concentration equal to $35 \%$. b) Increasing the part of flow curves of material II mixtures at two different solid concentrations. The empty circles are the flow curve at a solid concentration equal to $32 \%$, while the empty triangles are the flow curve at a solid concentration equal to $35 \%$. The dashed line is associated with the critical value of the shear rate for each material mixture tested.

\subsection{Sweep test results}

Fig. 4a shows the experimental results for the flow curves of two mixtures of material I at different solid concentrations. Once again, two different trends have been observed. In some cases (fig. 4 a for $35 \%$ solid concentration), there is first an increase in the stress with the shear rate at low shear stresses. This in fact corresponds to the response of the material in the solid regime. The rest of the flow curve is supposed to correspond to the material behaviour in the liquid regime: the transition to the liquid regime is associated with the rapid increase of the shear rate (stress plateau) above some critical value of the stress. At larger stresses, the curve slope increases. The plateau thus obtained for the stress increase is associated with the static yield stress, $\tau_{\mathrm{cl}}$, of the material (as observed in creep tests). Then the decreasing curve falls along the increasing curve. In other cases (fig. $4 \mathrm{a}$ for $40 \%$ solid concentration), the decreasing curve differs significantly from the increasing curve; there is a hysteresis where the stress for flow in the decreasing stress part is below the increasing curve. One can consider that the material was initially broken then liquefied, and the stress needed to maintain the flow is lower than the stress to break the initial structure. Under these conditions, the dynamic stress, $\tau_{\mathrm{c} 2}$, has been defined as the critical stress for flow stoppage when decreasing the stress level.

Generally, a material exhibiting a smooth solid-liquid transition (for example, material I at 35\% solid concentration, fig. 4a) has a good superposition of the increasing and decreasing stress curves, whereas those exhibiting an abrupt 
transition (for example, material I at 40\% solid concentration, fig. 4a) also exhibit the hysteresis in the flow curves. In the case of a smooth transition, the static yield stress is equal to the dynamic yield stress.

As mentioned above, the material mixtures tested exhibit a stress plateau in the flow curve at a particular stress value (i.e., the static yield stress), and the stress remains approximately constant in a certain range of shear rates. A typical example is shown in fig. $4 \mathrm{~b}$, which shows the flow curves of two mixtures of material II at different solid concentrations (32\% and 35\%) obtained with an increasing ramp of stress. It has been noted that when progressively increasing the stress level, a large increase of the resulting shear rate is observed around a critical value (the static yield stress), which rapidly transforms from a small value to a much larger value associated with the end of the plateau. This last value of the shear rate, called the critical shear rate, $\dot{\gamma}_{\mathrm{cr}}$, marks the transition of the material mixture behaviour from a yielding behaviour (low to high shear rate) to a steady state flow (high shear rate). In fact, no steady flows can be obtained below the critical shear rate $[12,13]$.

Considering that the liquid regime corresponds only to the decreasing stress curve, the Herschel-Bulkley rheological model has been used to fit it, which is a generalised model of a non-Newtonian fluid valid for yield stress fluids. The eqn. of the Herschel-Bulkley model is:

$$
\tau=\tau_{\mathrm{C} 2}+\mathrm{k} \dot{\gamma}^{\mathrm{n}}
$$

where $\dot{\gamma}$ is the shear rate, $\mathrm{k}$ is the consistent coefficient, and $\mathrm{n}$ is the flow index.

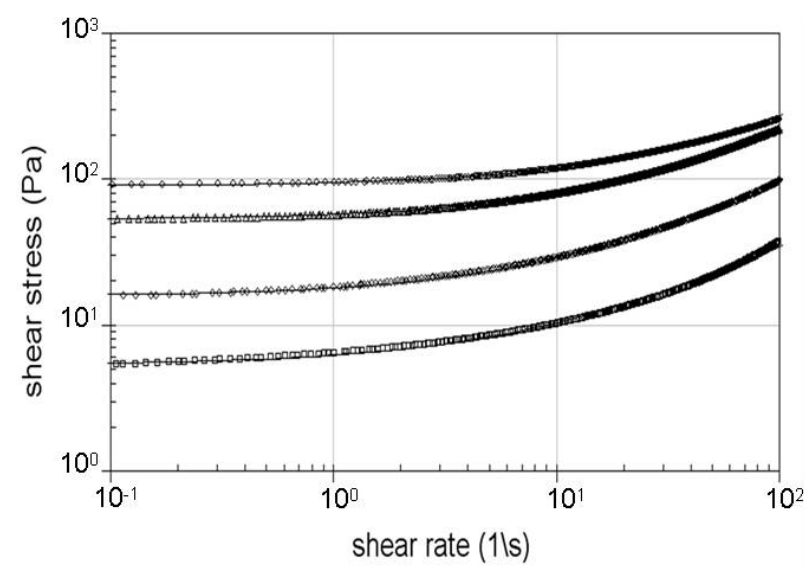

Figure 5: The experimental data (points) and the theoretical Herschel and Bulkley model (solid line) of material I at different solid volumetric concentration: the empty rectangles are the flow curves obtained at solid concentration of $35 \%$; the empty rhombuses are the flow curves obtained at solid concentration of $38 \%$; the empty triangles are the flow curves obtained at solid concentration of $40 \%$; and the empty circles are the flow curves obtained at solid concentration of $42 \%$. 
The consistency $\mathrm{k}$ is a simple constant of proportionality $\left[\mathrm{Pa} \cdot \mathrm{s}^{\mathrm{n}}\right]$. The dimensionless index $\mathrm{n}$ measures the degree to which the fluid is shear-thinning or shear-thickening [14].

In the ranges of solid concentration in which the materials mixtures analysed could be considered as homogeneous (32-42\% for material I and 30-38\% for material II), the debris flow material mixtures analysed behave like yield stress fluids. Fig. 6 shows the variation of the rheological parameters with the solid fraction. As mentioned before, it was found that the static yield stress (fig. 6a), the dynamic yield stress (fig. 6b) and the critical shear rate (fig. 6c) are higher with increasing solid fraction. An exponential function could be used to relate the rheological parameters to the solid volumetric concentration:

$$
\tau_{\mathrm{c} 1}, \tau_{\mathrm{C} 2}, \dot{\gamma}_{\mathrm{cr}}=\alpha \mathrm{e}^{\beta \Phi}
$$

where $\alpha$ and $\beta$ are fitting parameters, and their values are reported in table 2 .
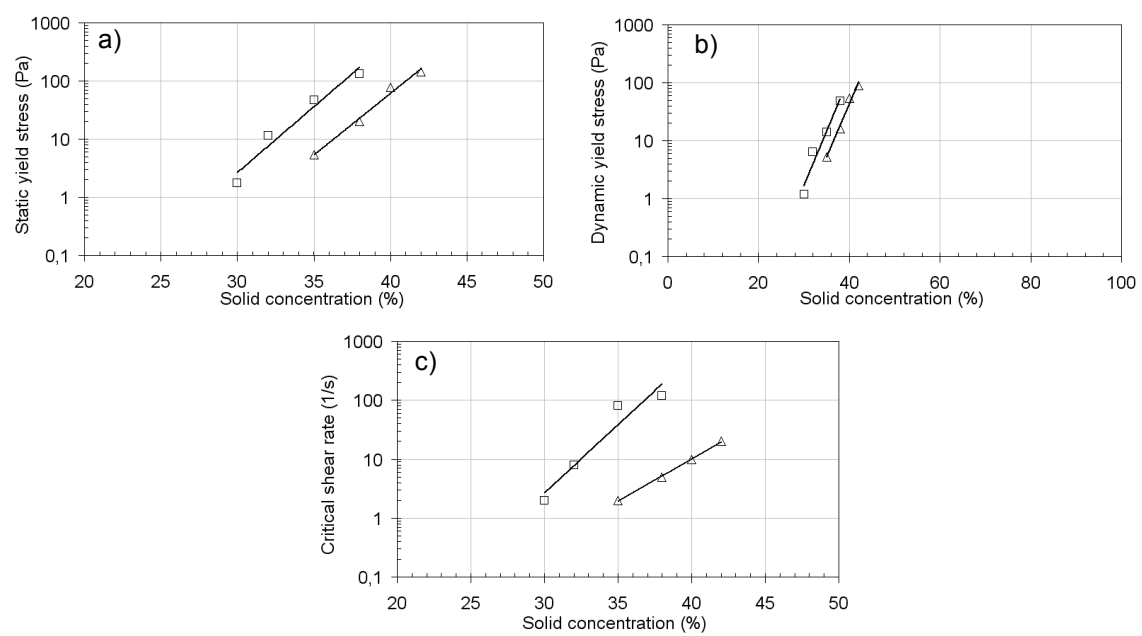

Figure 6: Influence of the solid concentration for the materials tested on: a) the static yield stress; b) the dynamic yield stress and c) the critical shear rate. The triangles are relative to material I and the rectangles are relative to material II.

Table 2: $\quad$ Fitting parameters $\alpha$ and $\beta$.

\begin{tabular}{|c|c|c|c|c|}
\hline & Material & $\alpha$ & $\beta$ & $\mathrm{R}^{2}$ \\
\hline \multirow{2}{*}{$\tau_{\mathrm{c} 1}$} & I & $2 \cdot 10^{-7}$ & 0,4874 & 0,987 \\
\cline { 2 - 5 } & II & $4 \cdot 10^{-7}$ & 0,5212 & 0,953 \\
\hline \multirow{2}{*}{$\tau_{\mathrm{c} 2}$} & I & $4 \cdot 10^{-6}$ & 0,4312 & 0,953 \\
\cline { 2 - 5 } & II & $2 \cdot 10^{-6}$ & 0,4196 & 0,983 \\
\hline \multirow{2}{*}{$\dot{\gamma}_{\mathrm{cr}}$} & I & $2 \cdot 10^{-5}$ & 0,3293 & 0,923 \\
\cline { 2 - 5 } & II & $3 \cdot 10^{-7}$ & 0,533 & 0,999 \\
\hline
\end{tabular}




\section{Inclined plane results}

The inclined plane allows measurement of the critical stress separating the solid and the liquid regime closer to the field conditions (i.e., flow over steep slopes) according to equations (4) and (5) reported in section 2. This test has been performed at the same solid volumetric concentration analysed with the rheometrical tests on materials I and II. The main results are that the dynamic and static yield stress increase with the solid concentration and that the static yield stress is always higher than the dynamic one. Both results are in agreement with the rheometrical ones. The comparison between the results of both tests is reported in fig. $7 \mathrm{a}$ for $\tau_{\mathrm{c} 1}$ and in fig. $7 \mathrm{~b}$ for $\tau_{\mathrm{c} 2}$. It has been observed that the materials analyzed show good agreement between the rheometrical results and the inclined plane results. This comparison suggests that the technique of the inclined plane might be used for determining the basic rheological parameters of materials including the whole range of particle sizes.
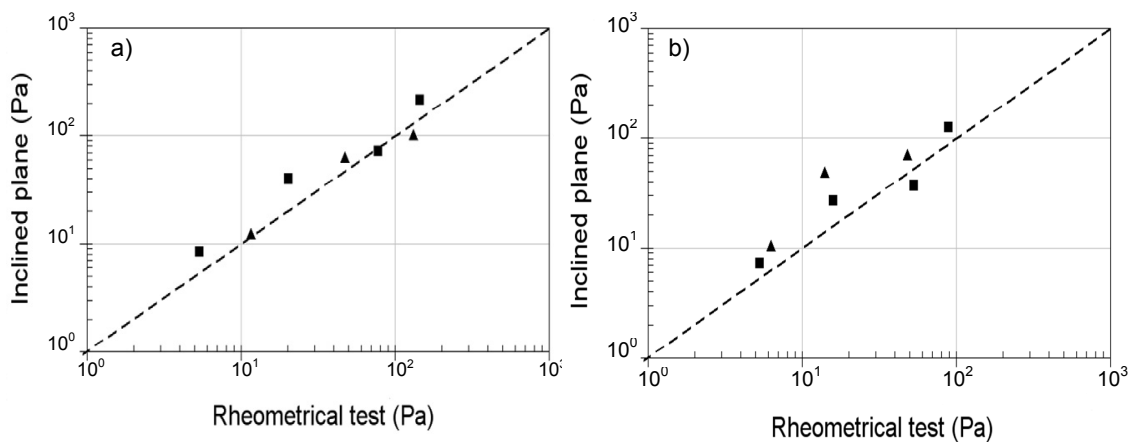

Figure 7: Comparison between the experimental results obtained from the inclined plane test and the experimental results obtained from the rheometrical test at different solid concentrations in terms of: a) static yield stress and b) dynamic yield stress. The filled triangles are relative to material I and the filled rectangles are relative to material II.

\section{Conclusion}

This paper concerns the study of the post-failure behaviour of pyroclastic debris flows in the Campania region (Italy). Two natural pyroclastic soils have been sampled and remixed with distilled water at different solid fractions. The behaviour of these mixtures has been investigated with a vane rotor rheometer and an incline plane. The principal result is the identification of a specific range of solid contents in which the mixtures could be considered as homogeneous fluid and could be tested with rheometrical apparatuses. Beyond these solid concentration ranges, the material mixtures behave like solids, while below 
them, the mixtures behave like pure liquids. For the materials analyzed the transition between "solid-like" to "fluid-like" behaviour occurs in a small range of solid concentrations and these ranges are quite different for each material, depending on the in situ porosity and the grain size distribution.

In the "fluid-like" behaviour the material mixtures behave like a yield stress fluid, and a classical Herschel and Bulkley model reproduces well the experimental data. Nevertheless, a hysteresis effect, associated with the instability of the material behaviour, has been observed for the high solid volumetric concentrations. The material starts to flow beyond a critical stress at a relatively large shear rate. These results suggest that in the field during the debris flow motion, a small variation of the solid fraction can lead to changing the behaviour from "solid-like" to "fluid-like" and vice versa. The critical shear rate related to low shear stress for lower solid fractions might explain the in situ observed post-failure behaviour of pyroclastic debris flows, which are able to flow over very long distances even over smooth slopes. Finally, the inclined plane technique could be used in the field for determining the static and dynamic yield stresses of natural pyroclastic suspensions.

\section{References}

[1] Cascini, L. \& Sorbino, G., The contribution of soil suction measurements to the analysis of flowslide triggering. Proc. Int. Workshop on Occurrence and Mechanisms of Flows in Natural Slopes and Earthfills - IW-Flows2003, Sorrento, pp 77-86, 2003.

[2] Scotto di Santolo, A., Le colate rapide. Helvelius Edizioni s.r.l., 2002.

[3] Scotto di Santolo, A., Analisi geotecnica dei fenomeni franosi nelle coltri piroclastiche della provincia di Napoli. PhD thesis, University of Naples "Federico II" and Rome "La Sapienza", 2000a.

[4] Scotto di Santolo, A., Analysis of a steep slope in unsaturated pyroclastic soils. Proc. Asian Conference on Unsaturated Soils, Singapore, pp 569-574, 2000b.

[5] Rickenmann, D. \& Koch, T., Comparison of debris flow modelling approaches. Proc. 1st Int. Conf. Debris flow Hazard Mitigation: Mechanics, Prediction, and Assessments, San Francisco, pp 576- 585, 1997.

[6] McDougall, S. \& Hungr, O., A model for the analysis of rapid landslide motion across three-dimensional terrain. Canadian Geotechnical Journal 41, pp 1084-1097, 2004.

[7] Revellino, P., Hungr, O., Guadagno, F.M. \& Evans, S.G., Velocity and runout prediction of destructive debris flows and debris avalanches in pyroclastic deposits, Campania region, Italy. Environmental Geology, 45, pp 295-311, 2004.

[8] Scotto di Santolo, A. \& Evangelista, A., Some observations on the prediction of the dynamic parameters of debris flows in pyroclastic deposits in the Campania region of Italy. Int. Journal of Natural Hazards 50, pp 605$622,2009$. 
62 Monitoring, Simulation, Prevention and Remediation of Dense and Debris Flows III

[9] Scotto di Santolo, A., Pellegrino, A. M. \& Evangelista, A., Experimental study on the rheological behaviour of debris flow material in Campania region, Fifth International Conference on Computational and Experimental Methods in Multiphase and Complex Flow, New Forest, pp 305-316 (2009).

[10] Papa, R., Indagine sperimentale di una copertura piroclastica di un versante della Campania, PhD thesis, University of Naples "Federico II", 2007.

[11] Van Wazer, R.J., Viscosity and flow measurement (a laboratory handbook of rheology). Interscience Publishers (New York), 1963.

[12] Coussot, P., Rheometry of Pastes, Suspensions and granular materials: Application in Industry and Environmental. A John Wiley \& Sons, Inc., Publications, 2005.

[13] Ovarlez, G., Rodts, S., Chateau, X. \& Coussot, P., Phenomenology and physical origin of shear localization of the shear banding in complex fluids. Rheologica Acta, 48, pp 831-844, 2009.

[14] Coussot, P., Mudflow Rheology and Dynamics, IAHR Monograph Series, A.A. Balkema: Rotterdam, 1997. 